Long-Term Care in Oklahoma - A 2021 Survey of Registered Voters 50 Years and Older: Annotated Questionnaire

February 2022

\title{
Screening Questions
}

S1. Our study is interested in the opinions of people in certain age groups. Could you please tell me your age as of your last birthday? [IN YEARS] [RECORD ACTUAL AGE AND USE THE AGE GROUPS BELOW TO KEEP TRACK OF HOW MANY RESPONDENTS WE ARE GETTING IN EACH GROUP] S2. [IF REFUSED IN S1:] I understand. Some people are not comfortable giving their age, but would you let me know which of the following age ranges you fall into?

\begin{tabular}{|l|c|}
\hline & Total \\
\hline Weighted $n$ & 1000 \\
\hline 50 to 54 & $15.5 \%$ \\
\hline 55 to 64 & $34.2 \%$ \\
\hline $65+$ & $50.2 \%$ \\
\hline Refused [THANK \& TERMINATE] & $0.0 \%$ \\
\hline
\end{tabular}

S3. To ensure it is recorded accurately, could you please state your gender? [CODE TO LIST BELOW]

\begin{tabular}{|l|c|}
\hline & Total \\
\hline Weighted $n$ & 1000 \\
\hline Man & $44.3 \%$ \\
\hline Woman & $55.4 \%$ \\
\hline Non-Binary & $0.1 \%$ \\
\hline Another gender identity & $0.1 \%$ \\
\hline Not sure [DO NOT READ] & $0.0 \%$ \\
\hline Refused [DO NOT READ] & $0.1 \%$ \\
\hline
\end{tabular}

S4. Are you registered to vote in Oklahoma?

\begin{tabular}{|l|c|}
\hline & Total \\
\hline Weighted $n$ & 1000 \\
\hline Yes & $100.0 \%$ \\
\hline No [THANK \& TERMINATE] & $0.0 \%$ \\
\hline
\end{tabular}


S5. What county do you live in? Code response to list below and note rural oversample counties.

\begin{tabular}{|l|c|}
\hline & Total \\
\hline Weighted $n$ & 1000 \\
\hline Urban counties & $69.4 \%$ \\
\hline Rural counties & $30.6 \%$ \\
\hline
\end{tabular}

\section{Main Questionnaire}

\section{$\underline{\text { Family Caregiver }}$}

Q1. A family caregiver is someone who provides UNPAID care for an adult loved one who is ill, frail, elderly, or has a physical, mental, or emotional disability. This UNPAID care may include assisting with personal needs like bathing and dressing, household chores, meals, shopping, transportation, or managing finances or medical care. Are you now or have you ever been a family caregiver who provided UNPAID care to an adult loved one? [INTERVIEWER NOTE: IF THE RESPONDENTS ASKS IF THE PERSON THEY PROVIDE OR HAVE PROVIDED CARE TO HAD TO LIVE WITH THEM, THE INTERVIEWER SHOULD SAY ... "THIIS PERSON DID NOT HAVE TO LIVE WITH YOU."]

\begin{tabular}{|l|c|c|}
\hline & Total & Current Caregivers \\
\hline Weighted $n$ & 1000 & 126 \\
\hline Yes, I am currently providing unpaid care to an adult loved one & $12.6 \%$ & $100.0 \%$ \\
\hline Yes, I have been an unpaid family caregiver in the past & $29.5 \%$ & $0.0 \%$ \\
\hline No, I have never been an unpaid caregiver & $57.4 \%$ & $0.0 \%$ \\
\hline Not sure [DO NOT READ] & $0.3 \%$ & $0.0 \%$ \\
\hline Refused [DO NOT READ] & $0.2 \%$ & $0.0 \%$ \\
\hline
\end{tabular}

Q2. IF CURRENT OR FORMER CAREGIVER, ASK Q2-Q7. Have you ever been employed either full- or part-time while providing care to your adult loved one?

\begin{tabular}{|l|c|c|}
\hline Current \& Former Caregivers & Total & Current Caregivers \\
\hline Weighted $n$ & 421 & 126 \\
\hline Yes & $57.8 \%$ & $53.5 \%$ \\
\hline No & $41.6 \%$ & $46.5 \%$ \\
\hline Not sure [DO NOT READ] & $0.5 \%$ & $0.0 \%$ \\
\hline Refused [DO NOT READ] & $0.0 \%$ & $0.0 \%$ \\
\hline
\end{tabular}


Q3. As a caregiver, have you ever incurred any expenses for the following services to help care for your loved one? [INSERT A-K IN RANDOM ORDER, BUT ALWAYS ASK A AND B TOGETHER]

\begin{tabular}{|c|c|c|}
\hline Current \& Former Caregivers & Total & Current Caregivers \\
\hline Weighted $n$ & 421 & 126 \\
\hline $\begin{array}{l}\text { a. General housing costs, such as rent, mortgage, utilities, and } \\
\text { other upkeep expenses }\end{array}$ & $43.0 \%$ & $47.8 \%$ \\
\hline $\begin{array}{l}\text { b. Making modifications to your home or a loved one's home to } \\
\text { help in their care }\end{array}$ & $40.9 \%$ & $47.7 \%$ \\
\hline $\begin{array}{l}\text { c. Hiring a home health aide or personal care attendant or other } \\
\text { in-home supportive services }\end{array}$ & $26.1 \%$ & $20.6 \%$ \\
\hline $\begin{array}{l}\text { d. Using respite [PRONOUNCED RES-PIT] care to get a break } \\
\text { from your caregiving responsibilities }\end{array}$ & $13.7 \%$ & $10.0 \%$ \\
\hline e. Using adult day care & $5.7 \%$ & $6.7 \%$ \\
\hline $\begin{array}{l}\text { f. Transporting your loved one to where they need to go, such } \\
\text { as to medical appointments }\end{array}$ & $84.2 \%$ & $83.0 \%$ \\
\hline g. Paying for nursing home or assisted living care & $13.7 \%$ & $10.9 \%$ \\
\hline $\begin{array}{l}\text { h. Purchasing equipment to help your loved one, such as a } \\
\text { wheelchair, hearing devices, vision aids, or wearable devices }\end{array}$ & $44.9 \%$ & $44.6 \%$ \\
\hline i. Purchasing prescription drugs & $49.2 \%$ & $51.3 \%$ \\
\hline j. Making modifications to a vehicle or buying a new vehicle & $17.1 \%$ & $25.8 \%$ \\
\hline $\begin{array}{l}\text { k. Paying for medical costs, such as direct payments to health } \\
\text { care providers, hospitals, or therapists }\end{array}$ & $32.4 \%$ & $37.0 \%$ \\
\hline
\end{tabular}

Q4. [ASK OF CURRENT CAREGIVERS ONLY] Thinking about the money you have spent out-of-your-own-pocket to care for your loved over the last year, including household expenses, medical expenses, personal care expenses, transportation, over-the-counter and prescription medications, and other expenses, what do you estimate you spent each month? [RECORD RESPONSE AS AN INDIVIDUAL VARIABLE AND ALSO CODE TO THE LIST BELOW]

\begin{tabular}{|l|c|}
\hline Current Caregivers & Total \\
\hline Weighted $n$ & 126 \\
\hline$\$ 0$ & $3.4 \%$ \\
\hline Less than $\$ 50$ & $7.1 \%$ \\
\hline$\$ 51$ to $\$ 99$ & $11.8 \%$ \\
\hline$\$ 101$ to $\$ 200$ & $6.6 \%$ \\
\hline$\$ 201$ to $\$ 300$ & $5.9 \%$ \\
\hline$\$ 301$ to $\$ 400$ & $2.6 \%$ \\
\hline$\$ 401$ to $\$ 500$ & $7.4 \%$ \\
\hline$\$ 501$ to $\$ 600$ & $0.8 \%$ \\
\hline$\$ 601$ to $\$ 700$ & $1.1 \%$ \\
\hline$\$ 701$ to $\$ 800$ & $1.8 \%$ \\
\hline$\$ 801$ to $\$ 900$ & $0.0 \%$ \\
\hline$\$ 901$ to $\$ 1,000$ & $5.8 \%$ \\
\hline Over $\$ 1,000$ a month & $8.0 \%$ \\
\hline Not sure [DO NOT READ] & $31.9 \%$ \\
\hline Refused [DO NOT READ] & $5.8 \%$ \\
\hline
\end{tabular}


Q5. IF A CURRENT CAREGIVER, ASK: Does the person you care for suffer from Alzheimer's, dementia, or other forms of mental confusion? IF A FORMER CAREGIVER, ASK: Did the person you cared for suffer from Alzheimer's, dementia, or other forms of mental confusion?

\begin{tabular}{|l|c|c|}
\hline Current \& Former Caregivers & Total & Current Caregivers \\
\hline Weighted $n$ & 421 & 126 \\
\hline Yes & $42.7 \%$ & $41.0 \%$ \\
\hline No & $56.3 \%$ & $58.0 \%$ \\
\hline Not sure [DO NOT READ] & $0.6 \%$ & $0.4 \%$ \\
\hline Refused [DO NOT READ] & $0.4 \%$ & $0.6 \%$ \\
\hline
\end{tabular}

Q6. Did the person you care [CARED FOR FORMER CAREGIVERS] for ever serve in the United States Armed Forces? This includes the Army, Navy, Air Force, Marines, Coast Guard, Women's Armed Forces, National Guard, or Reserves?

\begin{tabular}{|l|c|c|}
\hline Current \& Former Caregivers & Total & Current Caregivers \\
\hline Weighted $n$ & 421 & 126 \\
\hline Yes & $23.0 \%$ & $19.6 \%$ \\
\hline No & $76.1 \%$ & $79.8 \%$ \\
\hline Not sure [DO NOT READ] & $0.9 \%$ & $0.6 \%$ \\
\hline Refused [DO NOT READ] & $0.0 \%$ & $0.0 \%$ \\
\hline
\end{tabular}

Q7. As a caregiver do [DID FOR FORMER CAREGIVERS] you ever ...? [INSERT, READ AND RANDOMIZE ITEMS A-C] [RECORD A RESPONSE FOR EACH ITEM]

\begin{tabular}{|l|c|c|}
\hline Current \& Former Caregivers & Total & $\begin{array}{c}\text { Current } \\
\text { Caregivers }\end{array}$ \\
\hline Weighted $n$ & 421 & 126 \\
\hline a. Feel stressed emotionally due to your caregiving responsibilities & $74.0 \%$ & $67.6 \%$ \\
\hline b. Feel strained financially due to your caregiving responsibilities & $37.2 \%$ & $33.6 \%$ \\
\hline Weighted $n$ (working caregivers) & 243 & 67 \\
\hline $\begin{array}{l}\text { c. Have difficulties in balancing your job and family responsibilities } \\
\text { [SKIP IF ANSWERED NO, NOT SURE, OR REFUSED TO Q2] }\end{array}$ & $59.3 \%$ & $47.5 \%$ \\
\hline
\end{tabular}




\section{$\underline{\text { Respite Care }}$}

Q8. Oklahoma's respite care program provides short-term help from a home health aide or an adult day program so family caregivers can take a break from their caregiving duties. Do you support or oppose increasing access to this program so more family caregivers can use it? Q9. Is that strongly or somewhat?

\begin{tabular}{|l|c|c|c|c|}
\hline & Total & Republicans & Independents & Democrats \\
\hline Weighted $n$ & 1000 & 393 & 170 & 330 \\
\hline Strongly support & $69.2 \%$ & $63.3 \%$ & $69.8 \%$ & $80.9 \%$ \\
\hline Somewhat support & $17.1 \%$ & $22.4 \%$ & $14.2 \%$ & $13.1 \%$ \\
\hline $\begin{array}{l}\text { Neither support/oppose/not sure } \\
\text { [DO NOT READ] }\end{array}$ & $8.9 \%$ & $9.7 \%$ & $8.9 \%$ & $3.9 \%$ \\
\hline Somewhat oppose & $2.1 \%$ & $2.6 \%$ & $3.0 \%$ & $1.5 \%$ \\
\hline Strongly oppose & $2.5 \%$ & $2.0 \%$ & $4.1 \%$ & $0.6 \%$ \\
\hline Refused [DO NOT READ] & $0.2 \%$ & $0.0 \%$ & $0.0 \%$ & $0.0 \%$ \\
\hline SUPPORT & $86.3 \%$ & $85.7 \%$ & $84.0 \%$ & $94.0 \%$ \\
\hline OPPOSE & $4.6 \%$ & $4.6 \%$ & $7.1 \%$ & $2.1 \%$ \\
\hline
\end{tabular}

\section{$\underline{\text { Tax Credit }}$}

Q10. Do you support or oppose providing a state income tax credit to family caregivers who incur expenses for the care and support of a family member living in Oklahoma? Q11. Is that strongly or somewhat?

\begin{tabular}{|l|c|c|c|c|}
\hline & Total & Republicans & Independents & Democrats \\
\hline Weighted $n$ & 1000 & 393 & 170 & 330 \\
\hline Strongly support & $67.0 \%$ & $62.2 \%$ & $69.8 \%$ & $74.5 \%$ \\
\hline Somewhat support & $18.1 \%$ & $21.7 \%$ & $18.3 \%$ & $14.9 \%$ \\
\hline $\begin{array}{l}\text { Neither support/oppose/ } \\
\text { not sure [DO NOT READ] }\end{array}$ & $7.1 \%$ & $8.0 \%$ & $6.5 \%$ & $4.8 \%$ \\
\hline Somewhat oppose & $3.0 \%$ & $3.3 \%$ & $2.4 \%$ & $3.0 \%$ \\
\hline Strongly oppose & $4.8 \%$ & $4.8 \%$ & $3.0 \%$ & $2.7 \%$ \\
\hline Refused [DO NOT READ] & $0.1 \%$ & $0.0 \%$ & $0.0 \%$ & $0.0 \%$ \\
\hline SUPPORT & $85.1 \%$ & $83.9 \%$ & $88.1 \%$ & $89.4 \%$ \\
\hline OPPOSE & $7.8 \%$ & $8.1 \%$ & $5.4 \%$ & $5.7 \%$ \\
\hline
\end{tabular}




\section{Long-Term Care}

Long-term care assists older Americans who have chronic or acute conditions, disabilities, or frailties with the basic tasks of daily living that they can no longer handle on their own. This includes things like bathing and dressing, preparing meals, helping with medication and nursing tasks like wound care, paying bills, housekeeping, and helping with trips to the doctor or store. Long-term care can be provided at home or in a facility, like a nursing home.

Q12. If you or a loved one needed long-term care, where would you prefer to receive that help? [INSERT, READ AND RANDOMIZE ITEMS A-C. ACCEPT ONLY ONE ANSWER]

\begin{tabular}{|l|c|}
\hline & Total \\
\hline Weighted $n$ & 1000 \\
\hline a. At home with caregiver assistance & $86.0 \%$ \\
\hline b. In a nursing home & $2.6 \%$ \\
\hline c. In an assisted living facility or group home & $6.8 \%$ \\
\hline Something else [DO NOT READ] & $1.4 \%$ \\
\hline Not sure [DO NOT READ] & $3.2 \%$ \\
\hline Refused [DO NOT READ] & $0.0 \%$ \\
\hline
\end{tabular}

Q13. If you or a loved one needed long-term care, how important would it be to have services that would help you or your loved one stay at home for as long as possible? Is it ... [INSERT AND READ RESPONSE OPTIONS IN ORDER]?

\begin{tabular}{|l|c|}
\hline & Total \\
\hline Weighted $n$ & 1000 \\
\hline Extremely important & $65.3 \%$ \\
\hline Very important & $26.4 \%$ \\
\hline Somewhat important & $5.3 \%$ \\
\hline Not very important & $0.9 \%$ \\
\hline Not at all important & $1.1 \%$ \\
\hline Not sure [DO NOT READ] & $0.8 \%$ \\
\hline Refused [DO NOT READ] & $0.3 \%$ \\
\hline
\end{tabular}

Q14. If you or a loved one needed long-term care, how important is it for you or your loved one to be able to choose where those services are provided? Is it ... [INSERT AND READ RESPONSE OPTIONS IN ORDER]?

\begin{tabular}{|l|c|}
\hline & Total \\
\hline Weighted $n$ & 1000 \\
\hline Extremely important & $62.0 \%$ \\
\hline Very important & $31.9 \%$ \\
\hline Somewhat important & $4.2 \%$ \\
\hline Not very important & $0.5 \%$ \\
\hline Not at all important & $0.6 \%$ \\
\hline Not sure [DO NOT READ] & $0.6 \%$ \\
\hline Refused [DO NOT READ] & $0.1 \%$ \\
\hline
\end{tabular}


Nursing Homes

Q15. How important is it for nursing home residents to receive quality care? Is it ... [INSERT AND READ RESPONSE OPTIONS IN ORDER]?

\begin{tabular}{|l|c|}
\hline & Total \\
\hline Weighted $n$ & 1000 \\
\hline Extremely important & $74.2 \%$ \\
\hline Very important & $23.2 \%$ \\
\hline Somewhat important & $1.4 \%$ \\
\hline Not very important & $0.2 \%$ \\
\hline Not at all important & $0.3 \%$ \\
\hline Not sure [DO NOT READ] & $0.4 \%$ \\
\hline Refused [DO NOT READ] & $0.3 \%$ \\
\hline
\end{tabular}

Q16. If you or a loved one needed to receive care in a nursing home, how concerned would you be about ... [INSERT, READ AND RANDOMIZE ITEMS A-F]? Would you be ... [INSERT AND READ RESPONSE OPTIONS IN ORDER]

a. The cost of care

\begin{tabular}{|l|c|}
\hline \multicolumn{1}{|l|}{} & Total \\
\hline Weighted $n$ & 1000 \\
\hline Extremely concerned & $55.5 \%$ \\
\hline Very concerned & $30.1 \%$ \\
\hline Somewhat concerned & $9.1 \%$ \\
\hline Not very concerned & $1.6 \%$ \\
\hline Not at all concerned & $2.1 \%$ \\
\hline Not sure [DO NOT READ] & $1.2 \%$ \\
\hline Refused [DO NOT READ] & $0.4 \%$ \\
\hline
\end{tabular}

b. Exposure to COVID-19 or its variants or other infections

\begin{tabular}{|l|c|}
\hline & Total \\
\hline Weighted $n$ & 1000 \\
\hline Extremely concerned & $47.8 \%$ \\
\hline Very concerned & $25.0 \%$ \\
\hline Somewhat concerned & $13.8 \%$ \\
\hline Not very concerned & $5.7 \%$ \\
\hline Not at all concerned & $5.6 \%$ \\
\hline Not sure [DO NOT READ] & $1.4 \%$ \\
\hline Refused [DO NOT READ] & $0.6 \%$ \\
\hline
\end{tabular}


c. Being isolated and alone

\begin{tabular}{|l|c|}
\hline & Total \\
\hline Weighted $n$ & 1000 \\
\hline Extremely concerned & $54.8 \%$ \\
\hline Very concerned & $30.4 \%$ \\
\hline Somewhat concerned & $7.5 \%$ \\
\hline Not very concerned & $3.0 \%$ \\
\hline Not at all concerned & $2.8 \%$ \\
\hline Not sure [DO NOT READ] & $1.0 \%$ \\
\hline Refused [DO NOT READ] & $0.4 \%$ \\
\hline
\end{tabular}

d. Not being able to have visitors

\begin{tabular}{|l|c|}
\hline & Total \\
\hline Weighted $n$ & 1000 \\
\hline Extremely concerned & $55.1 \%$ \\
\hline Very concerned & $30.4 \%$ \\
\hline Somewhat concerned & $9.2 \%$ \\
\hline Not very concerned & $2.4 \%$ \\
\hline Not at all concerned & $1.2 \%$ \\
\hline Not sure [DO NOT READ] & $1.3 \%$ \\
\hline Refused [DO NOT READ] & $0.4 \%$ \\
\hline
\end{tabular}

e. Being abused or neglected

\begin{tabular}{|l|c|}
\hline & Total \\
\hline Weighted $n$ & 1000 \\
\hline Extremely concerned & $63.5 \%$ \\
\hline Very concerned & $24.5 \%$ \\
\hline Somewhat concerned & $7.0 \%$ \\
\hline Not very concerned & $2.1 \%$ \\
\hline Not at all concerned & $1.8 \%$ \\
\hline Not sure [DO NOT READ] & $0.6 \%$ \\
\hline Refused [DO NOT READ] & $0.5 \%$ \\
\hline
\end{tabular}

f. The nursing home not having enough staff to provide care

\begin{tabular}{|l|c|}
\hline & Total \\
\hline Weighted $n$ & 1000 \\
\hline Extremely concerned & $62.5 \%$ \\
\hline Very concerned & $29.2 \%$ \\
\hline Somewhat concerned & $5.5 \%$ \\
\hline Not very concerned & $0.3 \%$ \\
\hline Not at all concerned & $1.3 \%$ \\
\hline Not sure [DO NOT READ] & $0.9 \%$ \\
\hline Refused [DO NOT READ] & $0.4 \%$ \\
\hline
\end{tabular}


$\underline{\text { Transparency }}$

Q17. Currently, nursing homes and other long-term care facilities are generally required to be inspected once each year. Do you support or oppose increasing the frequency of these inspections to more than once each year? Q18. Is that strongly or somewhat?

\begin{tabular}{|l|c|c|c|c|}
\hline & Total & Republicans & Independents & Democrats \\
\hline Weighted $n$ & 1000 & 393 & 170 & 330 \\
\hline Strongly support & $82.9 \%$ & $81.7 \%$ & $87.6 \%$ & $85.4 \%$ \\
\hline Somewhat support & $8.4 \%$ & $8.1 \%$ & $7.7 \%$ & $7.9 \%$ \\
\hline $\begin{array}{l}\text { Neither support/oppose/not sure } \\
\text { [DO NOT READ] }\end{array}$ & $3.7 \%$ & $3.5 \%$ & $1.8 \%$ & $3.3 \%$ \\
\hline Somewhat oppose & $3.1 \%$ & $4.6 \%$ & $2.4 \%$ & $2.1 \%$ \\
\hline Strongly oppose & $1.4 \%$ & $1.5 \%$ & $0.6 \%$ & $1.2 \%$ \\
\hline Refused [DO NOT READ] & $0.5 \%$ & $0.5 \%$ & $0.0 \%$ & $0.0 \%$ \\
\hline SUPPORT & $91.3 \%$ & $89.8 \%$ & $95.3 \%$ & $93.3 \%$ \\
\hline OPPOSE & $4.5 \%$ & $6.1 \%$ & $3.0 \%$ & $3.3 \%$ \\
\hline
\end{tabular}

Q19. Do you support or oppose the Oklahoma legislature requiring more licensed inspectors to be available to inspect and evaluate the quality of care that nursing homes and other long-term care facilities are providing its residents in Oklahoma? Q20. Is that strongly or somewhat?

\begin{tabular}{|l|c|c|c|c|}
\hline & Total & Republicans & Independents & Democrats \\
\hline Weighted $n$ & 1000 & 393 & 170 & 330 \\
\hline Strongly support & $79.8 \%$ & $76.1 \%$ & $82.4 \%$ & $86.9 \%$ \\
\hline Somewhat support & $11.2 \%$ & $14.8 \%$ & $11.2 \%$ & $6.4 \%$ \\
\hline $\begin{array}{l}\text { Neither support/oppose/not sure } \\
\text { [DO NOT READ] }\end{array}$ & $5.7 \%$ & $5.9 \%$ & $4.7 \%$ & $4.5 \%$ \\
\hline Somewhat oppose & $1.3 \%$ & $1.8 \%$ & $1.2 \%$ & $0.6 \%$ \\
\hline Strongly oppose & $1.7 \%$ & $1.3 \%$ & $0.6 \%$ & $1.5 \%$ \\
\hline Refused [DO NOT READ] & $0.4 \%$ & $0.3 \%$ & $0.0 \%$ & $0.0 \%$ \\
\hline SUPPORT & $91.0 \%$ & $90.9 \%$ & $93.6 \%$ & $93.3 \%$ \\
\hline OPPOSE & $3.0 \%$ & $3.1 \%$ & $1.8 \%$ & $2.1 \%$ \\
\hline
\end{tabular}

Questions 21-26 may be released at a later date. 
Home Care

Q27. Do you support or oppose increasing home and community-based services to help seniors live independently at home longer as they age? Q28. Is that strongly or somewhat?

\begin{tabular}{|l|c|c|c|c|}
\hline & Total & Republicans & Independents & Democrats \\
\hline Weighted $n$ & 1000 & 393 & 170 & 330 \\
\hline Strongly support & $85.8 \%$ & $84.5 \%$ & $84.0 \%$ & $91.2 \%$ \\
\hline Somewhat support & $9.2 \%$ & $10.7 \%$ & $14.2 \%$ & $4.8 \%$ \\
\hline $\begin{array}{l}\text { Neither support/oppose/not sure } \\
\text { [DO NOT READ] }\end{array}$ & $2.4 \%$ & $2.1 \%$ & $0.6 \%$ & $2.4 \%$ \\
\hline Somewhat oppose & $0.8 \%$ & $1.3 \%$ & $0.0 \%$ & $0.3 \%$ \\
\hline Strongly oppose & $1.5 \%$ & $1.5 \%$ & $1.2 \%$ & $0.6 \%$ \\
\hline Refused [DO NOT READ] & $0.4 \%$ & $0.0 \%$ & $0.0 \%$ & $0.6 \%$ \\
\hline SUPPORT & $95.0 \%$ & $95.2 \%$ & $98.2 \%$ & $96.0 \%$ \\
\hline OPPOSE & $2.3 \%$ & $2.8 \%$ & $1.2 \%$ & $0.9 \%$ \\
\hline
\end{tabular}

Q29. The Oklahoma Legislature passed a law to create a program called Options Counseling to assist family caregivers with guidance, information, and resources to help them provide care to their loved ones. To date, this law has not been implemented. Do you support or oppose making sure that the state agency tasked with implementation of Options Counseling launches this program? Q30. Is that strongly or somewhat?

\begin{tabular}{|l|c|c|c|c|}
\hline & Total & Republicans & Independents & Democrats \\
\hline Weighted $n$ & 1000 & 393 & 170 & 330 \\
\hline Strongly support & $73.7 \%$ & $71.4 \%$ & $74.0 \%$ & $81.0 \%$ \\
\hline Somewhat support & $14.5 \%$ & $17.1 \%$ & $17.8 \%$ & $9.7 \%$ \\
\hline $\begin{array}{l}\text { Neither support/oppose/not sure } \\
\text { [DO NOT READ] }\end{array}$ & $7.5 \%$ & $7.4 \%$ & $4.8 \%$ & $5.7 \%$ \\
\hline Somewhat oppose & $1.8 \%$ & $1.5 \%$ & $0.6 \%$ & $2.7 \%$ \\
\hline Strongly oppose & $2.3 \%$ & $2.6 \%$ & $3.0 \%$ & $0.6 \%$ \\
\hline Refused [DO NOT READ] & $0.4 \%$ & $0.0 \%$ & $0.0 \%$ & $0.3 \%$ \\
\hline SUPPORT & $88.2 \%$ & $88.5 \%$ & $91.8 \%$ & $90.7 \%$ \\
\hline OPPOSE & $4.1 \%$ & $4.1 \%$ & $3.6 \%$ & $3.3 \%$ \\
\hline
\end{tabular}

Questions 31-47 may be released at a later date. 


\section{OAARP}

\section{Demographic Questions}

The following questions are for classification purposes only and will be kept entirely confidential.

D1. What is your current marital status? Are you ...? [INSERT AND READ EACH ANSWER CATEGORY]

\begin{tabular}{|l|c|}
\hline & Total \\
\hline Weighted $n$ & 1000 \\
\hline Married & $58.8 \%$ \\
\hline Not married, but living with your partner or significant other & $3.2 \%$ \\
\hline Separated & $0.6 \%$ \\
\hline Divorced & $10.1 \%$ \\
\hline Widowed & $14.6 \%$ \\
\hline Or are you currently single and never married & $8.0 \%$ \\
\hline Not sure [DO NOT READ] & $0.7 \%$ \\
\hline Refused [DO NOT READ] & $4.0 \%$ \\
\hline
\end{tabular}

D2. [IF D1 = 1 ASK: "Are you or your spouse currently a member of A-A-R-P?" IF D1 = 2 ASK: "Are you or your partner currently a member of A-A-R-P?" OTHERWISE ASK "Are you currently a member of A-A-R-P?"]

\begin{tabular}{|l|c|}
\hline & Total \\
\hline Weighted $n$ & 1000 \\
\hline Yes & $27.0 \%$ \\
\hline No & $67.0 \%$ \\
\hline Not sure [DO NOT READ] & $2.9 \%$ \\
\hline Refused [DO NOT READ] & $3.1 \%$ \\
\hline
\end{tabular}

D3. Would you say your health in general is ...? [INSERT AND READ EACH ANSWER CATEGORY]

\begin{tabular}{|l|c|}
\hline & Total \\
\hline Weighted $n$ & 1000 \\
\hline Excellent & $12.7 \%$ \\
\hline Very good & $31.0 \%$ \\
\hline Good & $33.6 \%$ \\
\hline Fair & $13.5 \%$ \\
\hline Poor & $4.7 \%$ \\
\hline Not sure [DO NOT READ] & $1.4 \%$ \\
\hline Refused [DO NOT READ] & $3.2 \%$ \\
\hline
\end{tabular}


D4. Are you of Hispanic, Spanish, or Latino origin or descent?

\begin{tabular}{|l|c|}
\hline & Total \\
\hline Weighted $n$ & 1000 \\
\hline Yes & $3.1 \%$ \\
\hline No & $92.6 \%$ \\
\hline Not sure [DO NOT READ] & $1.2 \%$ \\
\hline Refused [DO NOT READ] & $3.2 \%$ \\
\hline
\end{tabular}

D5. What is your race? Are you ...? [INSERT AND READ EACH ANSWER CATEGORY AND ACCEPT MULTIPLE RESPONSES]

\begin{tabular}{|l|c|}
\hline & Total \\
\hline Weighted $n$ & 1000 \\
\hline White or Caucasian & $80.4 \%$ \\
\hline Black or African American & $9.4 \%$ \\
\hline Native American or Alaskan Native & $6.1 \%$ \\
\hline Asian & $0.5 \%$ \\
\hline Native Hawaiian or other Pacific Islander & $0.4 \%$ \\
\hline Or are you some other race? & $1.8 \%$ \\
\hline Not sure [DO NOT READ] & $0.8 \%$ \\
\hline Refused [DO NOT READ] & $3.6 \%$ \\
\hline
\end{tabular}

D6. Do you own or rent your primary residence?

\begin{tabular}{|l|c|}
\hline & Total \\
\hline Weighted $n$ & 1000 \\
\hline Own & $82.3 \%$ \\
\hline Rent & $9.2 \%$ \\
\hline Other living arrangement [DO NOT READ] & $2.3 \%$ \\
\hline Not sure [DO NOT READ] & $1.6 \%$ \\
\hline Refused [DO NOT READ] & $4.6 \%$ \\
\hline
\end{tabular}

D7. What type of home is your primary residence? Is it ...? [READ EACH ANSWER CATEGORY]

\begin{tabular}{|l|c|}
\hline & Total \\
\hline Weighted $n$ & 1000 \\
\hline Single family home & $82.7 \%$ \\
\hline Mobile home & $5.9 \%$ \\
\hline Town home or duplex & $1.4 \%$ \\
\hline Apartment & $3.1 \%$ \\
\hline Condominium or coop, or & $0.6 \%$ \\
\hline Something else [DO NOT READ] & $1.2 \%$ \\
\hline Not sure [DO NOT READ] & $1.1 \%$ \\
\hline Refused [DO NOT READ] & $4.0 \%$ \\
\hline
\end{tabular}


D8. What is the highest level of education that you completed? Is it ...? [READ EACH ANSWER CATEGORY]

\begin{tabular}{|l|c|}
\hline & Total \\
\hline Weighted $n$ & 1000 \\
\hline 0 to $12^{\text {th }}$ grade, but with no diploma & $3.6 \%$ \\
\hline High school graduate or equivalent & $25.0 \%$ \\
\hline $\begin{array}{l}\text { Post high school education, but with no } \\
\text { degree }\end{array}$ & $15.2 \%$ \\
\hline 2 year degree & $16.2 \%$ \\
\hline 4 year degree & $14.7 \%$ \\
\hline Post graduate study, but with no degree & $6.1 \%$ \\
\hline Graduate or professional degree & $13.1 \%$ \\
\hline Not sure [DO NOT READ] & $1.6 \%$ \\
\hline Refused [DO NOT READ] & $4.4 \%$ \\
\hline
\end{tabular}

D9. Which of the following best describes your current employment status? Are you ...? [READ EACH ANSWER CATEGORY]

\begin{tabular}{|l|c|}
\hline & Total \\
\hline Weighted $n$ & 1000 \\
\hline Self-employed full-time & $7.1 \%$ \\
\hline Self-employed part-time & $2.2 \%$ \\
\hline Employed full-time & $22.0 \%$ \\
\hline Employed part-time & $4.3 \%$ \\
\hline Retired and not working at all & $52.4 \%$ \\
\hline Unemployed and looking for work & $1.5 \%$ \\
\hline $\begin{array}{l}\text { Or are you not in the labor force for other } \\
\text { reasons }\end{array}$ & $4.5 \%$ \\
\hline Not sure [DO NOT READ] & $1.6 \%$ \\
\hline Refused [DO NOT READ] & $4.5 \%$ \\
\hline
\end{tabular}

D10. Thinking about your state elections for Oklahoma Governor and State Legislators in the last 10 years, which of the following best describes your voting behavior? Would you say you vote ...? [INSERT AND READ EACH ANSWER CATEGORY]

\begin{tabular}{|l|c|}
\hline & Total \\
\hline Weighted $n$ & 1000 \\
\hline Always & $67.0 \%$ \\
\hline Most of the time & $22.0 \%$ \\
\hline About half of the time & $3.9 \%$ \\
\hline Seldom, or & $1.2 \%$ \\
\hline Never & $0.8 \%$ \\
\hline Not sure [DO NOT READ] & $1.3 \%$ \\
\hline Refused [DO NOT READ] & $3.8 \%$ \\
\hline
\end{tabular}


D11. [IF D10 <> NEVER, NOT SURE OR REFUSED, ASK D11] About how often do you vote in primary elections? These are elections by the political parties to nominate a candidate for the general election. Do you vote ... [INSERT AND READ EACH RESPONSE OPTION IN ORDER]?

\begin{tabular}{|l|c|}
\hline Has Voted in Last 10 Years & Total \\
\hline Weighted $n$ & 941 \\
\hline Always & $67.0 \%$ \\
\hline Most of the time & $20.9 \%$ \\
\hline About half of the time & $5.7 \%$ \\
\hline Seldom, or & $2.7 \%$ \\
\hline Never & $1.6 \%$ \\
\hline Not sure [DO NOT READ] & $0.4 \%$ \\
\hline Refused [DO NOT READ] & $1.7 \%$ \\
\hline
\end{tabular}

D12. Do you consider yourself to be a ...? [INSERT, READ AND RANDOMIZE ITEMS A-C, FOLLOW UP WITH D]

\begin{tabular}{|l|c|}
\hline \multicolumn{1}{|l|}{ Weighted $n$} & Total \\
\hline a. Democrat & 1000 \\
\hline b. Republican & $33.0 \%$ \\
\hline c. Independent & $39.3 \%$ \\
\hline d. Something else [DO NOT READ] & $17.0 \%$ \\
\hline Not sure [DO NOT READ] & $2.0 \%$ \\
\hline Refused [DO NOT READ] & $2.7 \%$ \\
\hline
\end{tabular}

D13. How would you characterize your political views? Would you say you are ...? [INSERT, READ AND RANDOMIZE ITEMS A-C]

\begin{tabular}{|l|c|}
\hline & Total \\
\hline Weighted $n$ & 1000 \\
\hline a. Conservative & $44.1 \%$ \\
\hline b. Moderate & $30.5 \%$ \\
\hline c. Liberal & $13.6 \%$ \\
\hline None of these [DO NOT READ] & $1.4 \%$ \\
\hline Not sure [DO NOT READ] & $4.5 \%$ \\
\hline Refused [DO NOT READ] & $5.9 \%$ \\
\hline
\end{tabular}


D14. We realize income is a private matter and so rather than ask you anything specific about your income, l'd like to ask you to please stop me when I get to the category that includes your household's income before taxes in 2020. Was it ...? [INSERT AND READ EACH ANSWER CATEGORY]

\begin{tabular}{|l|c|}
\hline & Total \\
\hline Weighted $n$ & 1000 \\
\hline Less than $\$ 10,000$ & $3.1 \%$ \\
\hline$\$ 10,000$ to less than $\$ 20,000$ & $5.1 \%$ \\
\hline$\$ 20,000$ to less than $\$ 30,000$ & $6.8 \%$ \\
\hline$\$ 30,000$ to less than $\$ 40,000$ & $8.1 \%$ \\
\hline$\$ 40,000$ to less than $\$ 50,000$ & $10.8 \%$ \\
\hline$\$ 50,000$ to less than $\$ 60,000$ & $7.5 \%$ \\
\hline$\$ 60,000$ to less than $\$ 75,000$ & $8.2 \%$ \\
\hline$\$ 75,000$ to less than $\$ 100,000$ & $10.0 \%$ \\
\hline$\$ 100,000$ to less than $\$ 125,000$ & $4.8 \%$ \\
\hline$\$ 125,000$ to less than $\$ 150,000$ & $3.6 \%$ \\
\hline$\$ 150,000$ to less than $\$ 200,000$ & $2.3 \%$ \\
\hline$\$ 200,000$ or more & $2.7 \%$ \\
\hline Not sure [DO NOT READ] & $4.5 \%$ \\
\hline Refused [DO NOT READ] & $22.5 \%$ \\
\hline
\end{tabular}

D15. Which of the following best describes the community where you live? [INSERT AND READ EACH RESPONSE OPTION IN ORDER]

\begin{tabular}{|l|c|}
\hline & Total \\
\hline Weighted $n$ & 1000 \\
\hline An urban area near a mix of offices, apartments and shops & $6.9 \%$ \\
\hline An urban area that is mostly residential & $12.4 \%$ \\
\hline A suburban area with a mix of offices, apartments and shops & $5.5 \%$ \\
\hline A suburban area that is mostly residential & $17.3 \%$ \\
\hline A small town, or & $23.9 \%$ \\
\hline A rural area & $25.6 \%$ \\
\hline Not sure [DO NOT READ] & $3.9 \%$ \\
\hline Refused [DO NOT READ] & $4.5 \%$ \\
\hline
\end{tabular}

D16. Does any disability, handicap, or chronic disease keep you from participating fully in work, school, household, or other activities?

\begin{tabular}{|l|c|}
\hline & Total \\
\hline Weighted $n$ & 1000 \\
\hline Yes & $24.9 \%$ \\
\hline No & $69.4 \%$ \\
\hline Not sure [DO NOT READ] & $1.4 \%$ \\
\hline Refused [DO NOT READ] & $4.3 \%$ \\
\hline
\end{tabular}

Thank you. That is our last question for today/tonight. 


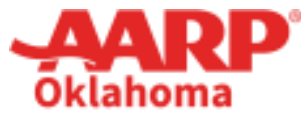

\section{Methodology}

AARP Oklahoma commissioned a telephone survey among 1,000 registered voters age 50-plus in Oklahoma to learn their views on long-term care and related proposals being considered by the Oklahoma State Legislature. The sample utilized an age-targeted landline and cell phone registered voter list obtained from Aristotle International. This study included an oversample of 200 registered voters who live outside of the Tulsa and Oklahoma MSAs and who didn't live in these counties: Comanche, Garfield, Muskogee, Pottawatomie, and Washington.

The sample database consisted of 997,621 registered voters age 50-plus in the State of Oklahoma. There are approximately 352,954 residents of Oklahoma age 50-plus who are not registered to vote, and there are approximately 430,276 registered voters age 50-plus who did not have a phone number on file that were not included in the sampling of this study.

Quotas were used in this study based on age and gender and were filled based on responses to questions in the questionnaire. They were designed to yield a proportional representation of the Oklahoma age 50-plus registered voter population. Respondents were selected at random from the sample list to be dialed. American Directions Research Group (ADRG) asked to speak with the registered voter listed on the file. If that person was unavailable, ADRG asked to speak with another member of the household aged 50-plus who was registered to vote in Oklahoma. The interviews averaged 21 minutes in length and were conducted in English. The survey has a margin of sampling error of \pm 3.1 percent.

This annotation highlights results from 1,000 registered voters interviewed from December 1 , 2021 to December 21, 2021. The data collection was performed by ADRG. The data is weighted by age, gender, AARP membership status, and geography. Percentages reported are rounded. For more information about the methodology, contact Terri Guengerich, AARP Research, tguengerich@aarp.org, (202)434-6306. 